\title{
Quantum Information Features Attendant to Atomic Spontaneous Decay
}

\author{
A. S. F. Obada ${ }^{1}$ S. Abdel-Khalek ${ }^{2,3}$ and M. E. Shaheen ${ }^{4, *}$ \\ ${ }^{1}$ Department of Mathematics, Faculty of Science, Al-Azhar University, Nasr City, Cairo, Egypt \\ 2 Department of Mathematics, Faculty of Science, Sohag University, 82524 Sohag, Egypt \\ ${ }^{3}$ Department of Mathematics, Faculty of Science, Taif University, Taif, Saudi Arabia \\ ${ }^{4}$ Department of Mathematics, Faculty of Science, Port Said University, Port Said, Egypt
}

Received: 30 May. 2013, Revised: 4 Oct. 2013, Accepted: 5 Oct. 2013

Published online: 1 May. 2014

\begin{abstract}
The effect of atomic spontaneous decay is considered for a two-level atom interacting with a single mode of electromagnetic field.The exact solution of the master equation is found for a chosen initial state. We study the effects of atomic decay on information and entanglement through temporal evolution of atomic quantum Fisher information, partial entropy of the atom and negativity.
\end{abstract}

Keywords: Spontaneous decay, Entanglement, Quantum Fisher information, Negativity.

\section{Introduction}

The Jaynes-Cummings (JC) model [1] has been used extensively in quantum optics to describe the quantum feature of the interaction of a single two-level atom with a quantized radiation field. Despite its simplicity, the JC model has great importance because technological advances have enabled the researchers to experimentally realize this rather idealized mode $[2,3,4]$. Excited by the success of the JC model, more researchers have paid special attention to generalize the model in order to investigate new quantum effects [5]. Such a generalization is of considerable interest because of its relevance to the study of the nonlinear coupling between a two-level atom and the radiation field $[6,7,8,9,10]$. Much work has been concentrated to the theoretical study of the dissipative JC model $[11,12,13]$ by considering cavities losses and atomic decay. Also, there are some experiments that have shown decoherence of the superposition of states due to the interaction of the system with the environment [14]. After introducing of the JC model, attention has been focused on some dissipative variants of this model in the last three decades $[15,16,17$, 18,19]. The experiments with highly excited Rydberg atoms allowed some of the predictions of the extended versions of the JC model to be observed. Besides the experimental drive, also there exists a theoretical motivation to include relevant damping mechanism to the JC model because its dynamics is more interesting. The dissipative effects of the JC model are caused by the energy exchange between the system and environment which is represented by a thermal reservoir. Since entanglement is a central topic in quantum information science, the degree of entanglement in some physical systems is studied [20]. Also there are many studies that focused on the properties of entanglement [21]-[29] which neglect the damping of the atom. However, the entanglement induced by the atomic damping of a dispersive reservoir was discussed in [30]. In this paper, we investigate the quantum feature attendant to atomic damping especially the entanglement. In the literature,there are more than one measure for entanglement such as atomic quantum Fisher information, von Neumman reduced entropy and negativity [30] . In this article, we find the exact solution of the master equation with atomic damping by using dressed-state (D-S) representation. the paper is arranged as follows: Section 2 is dedicated to JC model and solution of density matrix elements. In Section 3, we present our results and discussion of entanglement using various measures with focus on effects of atomic damping. Finally, in Section 4, we exhibit our conclusion.

\footnotetext{
*Corresponding author e-mail: marman_eg@yahoo.com
} 


\section{The model and solution of master equation}

Consider two-level atom interacting resonantly with a single mode of electromagnetic field with intensity dependent coupling and described by Hamiltonian [31]

$$
H=\omega\left(\frac{\hat{\sigma}_{z}}{2}+\hat{a}^{+} \hat{a}\right)+\lambda\left(\sqrt{\hat{a}^{+} \hat{a}} \hat{a}^{+} \hat{\sigma}_{-}+\hat{a} \sqrt{\hat{a}^{+} \hat{a}} \hat{\sigma}_{+}\right)
$$

where $\omega$ is the field frequency as well as the transition frequency of the two level tom interacting resonantly with the cavity field, $\lambda$ is the atom-field coupling constant, $\hat{\sigma}_{z}=|e\rangle\langle e|-| g\rangle\langle g|$ is the population inversion operator, $\hat{\sigma}_{ \pm}$are the Pauli raising and lowering operators and $\hat{a}^{+}, \hat{a}$ are the creation and the annihilation operators which describe the cavity field. The Hamiltonians (1) describes a nonlinear transition from $|e\rangle \leftrightarrow|g\rangle$ in the two level system through intensity dependent coupling. Now, we consider the master equation which describes only the atomic damping of thermal reservoir $[32,33,34]$ by the parameter $\gamma_{A}$, given by

$\frac{d \hat{\rho}(t)}{d t}=-i[\hat{H}, \hat{\rho}(t)]+\gamma_{A}\left(2 \hat{\sigma}_{-} \hat{\rho} \hat{\sigma}_{+}-\hat{\sigma}_{+} \hat{\sigma}_{-} \hat{\rho}-\hat{\rho} \hat{\sigma}_{+} \hat{\sigma}_{-}\right)$

The exact solution for this equation in the case of a high-Q cavity $(\gamma<<\lambda)$ is obtained by analytic method by using the D-S representation [34] i.e., representation consisting of the complete set of eigenstates of the Hamiltonian (1), which is given by

$$
\left|\beta_{n}^{ \pm}\right\rangle=\frac{1}{\sqrt{2}}(|e, n\rangle \pm|g, n+1\rangle) \quad(n=0,1,2, \ldots)
$$

with

$$
\begin{array}{rlrl}
H|g, 0\rangle=-\frac{\omega}{2}, & H\left|\beta_{n}^{ \pm}\right\rangle & =E_{n}{ }^{ \pm}\left|\beta_{n}^{ \pm}\right\rangle \\
{E_{n}}^{ \pm}=\omega\left(n+\frac{1}{2}\right) \pm \mu_{n} & \mu_{n}=\lambda(n+1)
\end{array}
$$

We write the atomic operators in terms of the D-S as

$$
\begin{aligned}
& \hat{\sigma}_{+}= \frac{\left.\left|\beta_{0}^{+}\right\rangle\langle g, 0|\right)+\left|\beta_{0}^{-}\right\rangle\langle g, 0|}{\sqrt{2}}+\sum_{n=1}^{\infty}\left|\beta_{n}^{+}\right\rangle\left\langle\beta_{n-1}^{+}\right| \\
&-\left|\beta_{n}^{+}\right\rangle\left\langle\beta_{n-1}^{-}|+| \beta_{n}^{-}\right\rangle\left\langle\beta_{n-1}^{+}|-| \beta_{n}^{-}\right\rangle\left\langle\beta_{n-1}^{-}\right| \\
& \hat{\sigma}_{+} \hat{\sigma}_{-}=\sum_{n=0}^{\infty}\left|\beta_{n}^{+}\right\rangle\left\langle\beta_{n}^{+}|+| \beta_{n}^{+}\right\rangle\left\langle\beta_{n}^{-}|+| \beta_{n}^{-}\right\rangle\left\langle\beta_{n}^{+}\right| \\
&+\left|\beta_{n}^{-}\right\rangle\left\langle\beta_{n}^{-}\right|
\end{aligned}
$$

and using the following representation

$$
\widehat{\dot{x}}=e^{i \hat{H} t} \frac{\partial \hat{x}(t)}{\partial t} e^{-i \hat{H} t}+i[\hat{H}, \hat{x}(t)]
$$

where $\hat{x}$ is an arbitrary operator of the combined system. We assume that the atom initially in its excited state i.e. $|e\rangle$, while the radiation field is in the coherent state which is defined as

$$
|\alpha\rangle=e^{-\frac{1}{2}|\alpha|^{2}} \sum_{n=0}^{\infty} \frac{\alpha^{n}}{\sqrt{n !}}|n\rangle=\sum_{n=0}^{\infty} q_{n}|n\rangle
$$

Therefore, $\hat{x}(0)$ in the D-S representation is given by

$$
\begin{aligned}
\hat{x}(0)= & \frac{1}{2} \sum_{j, k=0}^{\infty} q_{k} q_{j}^{*}\left(\left|\beta_{k}^{+}\right\rangle\left\langle\beta_{j}^{+}|+| \beta_{k}^{+}\right\rangle\left\langle\beta_{j}^{-}|+| \beta_{k}^{-}\right\rangle\left\langle\beta_{j}^{+}\right|\right. \\
& \left.-\left|\beta_{k}^{-}\right\rangle\left\langle\beta_{j}^{-}\right|\right) .
\end{aligned}
$$

The solution of the master equation in the high-Q limit with the considered initial condition

$$
\begin{aligned}
\hat{\rho}(t)= & \sum_{j, k=0}^{\infty}\left[\rho_{k, j}^{e e}(t)|k, e\rangle\left\langle j, e\left|+\rho_{k, j}^{e g}(t)\right| k, e\right\rangle\langle j+1, g|+\right. \\
& \left.\rho_{k, j}^{g e}(t)|k+1, g\rangle\left\langle j, e\left|+\rho_{k, j}^{g g}(t)\right| k+1, g\right\rangle\langle j+1, g|\right]
\end{aligned}
$$

where

$\rho_{k, j}^{e e}(t)=\left\{\begin{array}{cr}q_{k} q_{j}^{*} e^{-[\gamma+i \omega(k-j)] t} \cos \mu_{k} t \cos \mu_{j} t & \forall k \neq j \\ \frac{1}{2}\left(\chi_{k}\right)+\left|q_{k}\right|^{2} e^{-\gamma t} \cos 2 \mu_{k} t & \forall k=j\end{array}\right.$

$\rho_{k, j}^{e g}(t)=\left(\rho_{k, j}^{g e}(t)\right)^{+}=i q_{k} q_{j}^{*} e^{-[\gamma+i \omega(k-j)] t} \sin \mu_{j} t \cos \mu_{k} t \quad \forall k, j$

$\rho_{k, j}^{g g}(t)=\left\{\begin{array}{cc}q_{k} q_{j}^{*} e^{-[\gamma+i \omega(k-j)] t} \sin \mu_{k} t \sin \mu_{j} t & \forall k \neq j \\ \frac{1}{2}\left(\chi_{k}\right)-\left|q_{k}\right|^{2} e^{-\gamma t} \cos 2 \mu_{k} t & \forall k=j\end{array}\right.$

with

$\chi_{k}=e^{-(\bar{n}+\gamma t)}\left(\frac{\bar{n}}{\gamma t}\right)^{\frac{k}{2}} I_{k}(2 \sqrt{\bar{n} \gamma t})$

where $I_{k}(\cdot)$ is the modified Bessl function and $\bar{n}=|\alpha|^{2}$. The coherence properties and entanglement will be studied by using the exact solution (8)-(10).

In the following section we use the elements of density matrix to obtain information features of the system such as entanglement, total correlation and degree of mixing of the modified JCM model with atomic damping.

\section{Measures of entanglement}

We start by mentioning of the measures and indicators of entanglement. 


\section{1 von Neumman entropy}

We start our investigation with the degradation of purity of the system states caused by atomic damping reservoir, by using von Neumman entropy

$$
S_{T}=-\sum_{i=0}^{\infty} \lambda_{i}^{S} \ln \left(\lambda_{i}^{S}\right)
$$

which may be used as a measure of entanglement of the state of the whole system, where $\lambda_{i}^{S}$ are eigenvalues of the density operator of the system $\hat{\rho}(t)$.

The reduced entropy of the field and the atom have been introduced in $[35,36]$. It has been shown that the reduced entropy is very useful and sensitive measure of the purity of the quantum state, because it includes all the moments of the density operator. The purity of the field state investigates by using the field entropy $S_{F}$, which is defined by

$$
S_{F}=-\sum_{i=0}^{\infty} \lambda_{i}^{F} \ln \left(\lambda_{i}^{F}\right)
$$

where $\lambda_{i}^{F}$ are the eigenvalues of the reduced field density matrix $\hat{\rho}_{F}(t)=\operatorname{Tr}_{A}\{\hat{\rho}(t)\}$, which is computed by using numerical calculation. To study the purity loss of atomic state, we use the marginal density matrix of the atom $S_{A}$ which is given by

$$
\varepsilon_{A}=-\sum_{i=0}^{2} \lambda_{i}^{A} \ln \left(\lambda_{i}^{A}\right)
$$

where

$\lambda_{1,2}^{A}=\frac{1}{2}\left\{1 \pm \sqrt{\left(\sum_{i=0} \rho_{i}^{e e}(t)-\rho_{i}^{g g}(t)\right)^{2}+4\left|\sum_{i=0} \rho_{i}^{e g}(t)\right|^{2}}\right.$, and $\lambda_{1,2}^{A}$ are eigenvalues of the reduced density matrix $\hat{\rho}_{A}(t)=\operatorname{Tr}_{F}\{\hat{\rho}(t)\}$.

\subsection{Atomic quantum Fisher information (QFI)}

The QFI, which lies at the heart of the quantum estimation theory, is the information we know about a certain parameter in a quantum state. It is related to the degree of statistical distinguishability of a quantum state from its neighbors in parameter space [40,41]. Considering a quantum state $\rho(\theta)$ with $\theta$ a parameter, the QFI for $\theta$ is defined as

$$
F_{Q}(\theta)=\operatorname{tr}\left[\rho(\theta) L_{\theta}^{2}\right]
$$

where $L_{\theta}$, the so-called symmetric logarithmic derivative, is determined by the following equation

$$
\partial_{\theta} \rho(\theta)=\frac{1}{2}\left[L_{\theta} \rho(\theta)+\rho(\theta) L_{\theta}\right]
$$

where $\partial_{\theta}=\frac{\partial}{\partial \theta}$ and $L_{\theta}$ can be resolved by rewriting the above equation.

If we want to estimate $\theta$ through measuring $\rho(\theta)$, the variance of $\theta$ is limited by a fundamental bound, which is

given by the quantum Cramer-Rao (QCR)[42,43] inequality

$$
\triangle \theta \geqslant \triangle \theta_{Q C R}=\frac{1}{\sqrt{F_{Q}(\theta)}}
$$

If $F_{Q}(\theta)$ is large, the variance $(\triangle)$ of our estimation is small.

\subsection{Negativity}

Negativity is a good measure of entanglement in every sense. It a computable measure of entanglement and thereby fill an important gap in the study of entanglement. It is based on the trace norm of the partial transpose $\hat{\rho}^{T_{A}}$ of the bipartite mixed state $\rho$, a quantity whose evaluation is completely straightforward using standard linear algebra packages. It essentially measures the degree to which $\rho^{T_{A}}$ fails to be positive, and therefore it can be regarded as a quantitative version of Peres' criterion for separability[37]. From the trace norm of $\rho^{T_{A}}$, denoted by $\left\|\hat{\rho}^{T_{A}}\right\|_{1}$, the negativity is defined as

$$
N(\rho)=\frac{\left\|\hat{\rho}^{T_{A},}\right\|_{1}-1}{2}
$$

which corresponds to the absolute value of the sum of negative eigenvalues of $\hat{\rho}^{T_{A}}$ [38], and which vanishes for unentangled states, $N(\rho)$ does not increase under local operations and classical communications (LOCC), i.e., it is an entanglement monotone [39], and as such it can be used to quantify the degree of entanglement in composite systems.

In the following section we discuss the effect of damping parameter on entanglement properties through the previous measures.

\section{Discussion and results}

Now, we proceed to describe the Figures. In Fig. 1 we plot atomic von Neumman entropy $S_{A}(T)$ and atomic QFI $F_{A}(T)$ against scaled time $T=\lambda t$ for the atom initially in the excited state $|e\rangle$ and field is in the coherent state $|\alpha\rangle$ with $\alpha=4$ with different values of the atomic damping parameter $\frac{\gamma}{\lambda}$. From Fig. 1(a,b), we find that at weak damping $\frac{\gamma}{\lambda}=0.00001$ the entropy and QFI exhibit a periodic behaviour with period $(n \pi, n=1,2,3 \ldots)$ with unvariant amplitude, we notice that information about the atom is lost in Fig. 1(b) in wide range between every two local maxima. For larger value of damping parameter to 
$\frac{\gamma}{\lambda}=0.3$ in Fig.1(c,d), behaviour of entropy and QFI shows some oscillations but when time increases entropy tends to stationary behaviour and information is lost completely in Fig. 1(d). Increasing damping parameter makes atom goes finally $(t \rightarrow \infty)$ to maximally entangled state and information is lost completely in short time.

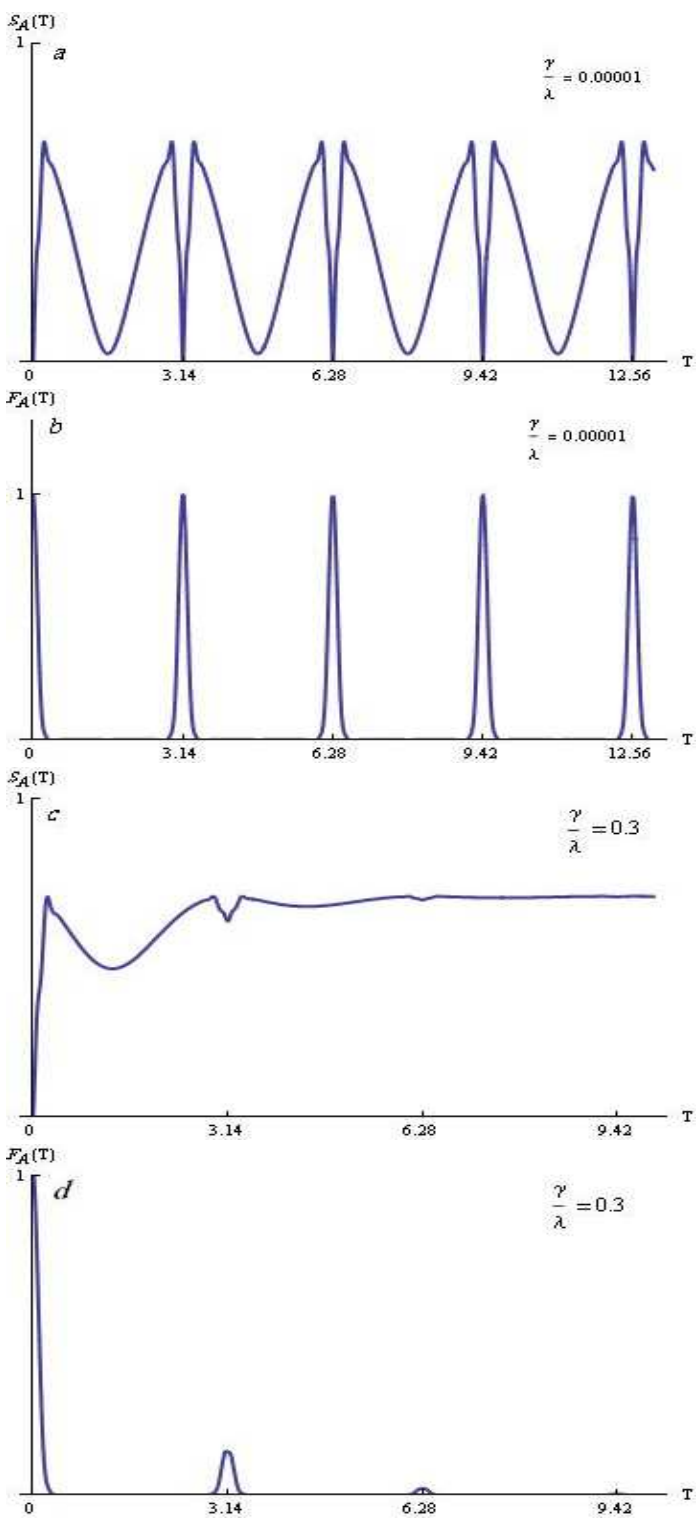

Fig. 1. The evolution of the: atomic von Neumman entropy

$S_{A}(T)$ and quantum Fisher information $F_{A}(T)$ for $\alpha=4$ with $\frac{\gamma}{\lambda}=0.00001$ in Fig.1 (a,b) and $\frac{\gamma}{\lambda}=0.3$ in Fig.1 (c,d)

To visualize the influence of atomic damping on the temporal evolution of the negativity and the von Neumman entropy. We have plotted $N(\rho)$ and $S(\rho)$ with different values of the atomic damping parameter $\frac{\gamma}{\lambda}=0.00001,0.3$ (see Fig. 2). From Fig. 2(a), the negativity with $\frac{\gamma}{\lambda}=0.00001$ behave similar to the correspondence atomic von Neumman entropy but with different scale maxima of $S_{A}(T)=\ln (2)$ and maxima of $N(\rho)=0.5$. For $\frac{\gamma}{\lambda}=0.3$, negativity exhibits oscillatory behaviour, the negativity $N(\rho)$ of the global system vanishes in the asymptotic limit, this means that in this region $(N(\rho)=0)$ the entanglement between the atom and the field is completely destroyed, and the total state of the system disentangled in a finite time and goes to a vacuum state. Finally, Fig. 2(c) presents the von Neumman entropy $S(\rho)$ of the total system for different previous values of the damping parameter. For weak damping parameter the total entropy $S(\rho)=0$ at any time since there is no dissipation almost, this means that the atom and the field are disentangled. For $\frac{\gamma}{\lambda}=0.3, S(\rho)$ arise rapidly as will as the coherence loss of the atom and the field. Finally the total state will reach a statistically mixed state and hence loss of information.
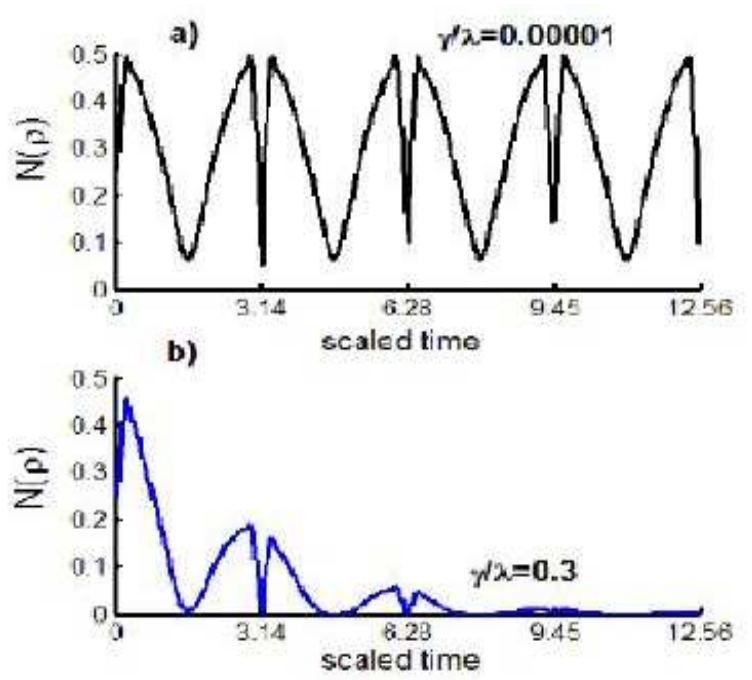

c)

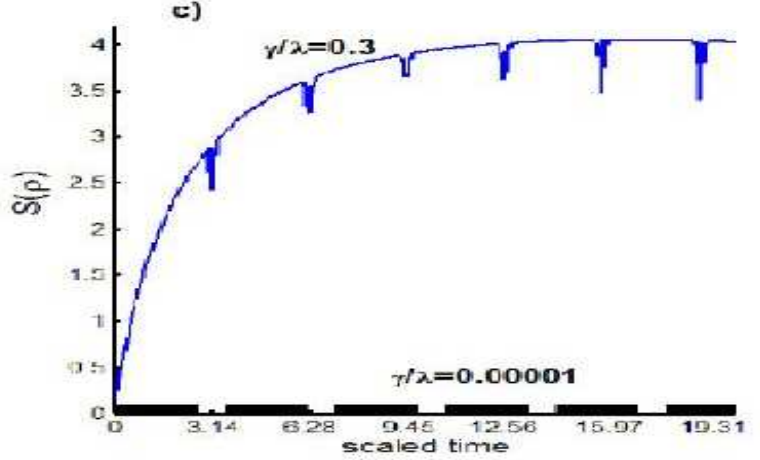

Fig. 2.The evolution total von Neumman entropy $S(\rho)$ and negativity $N(\rho)$ for $\alpha=4$ with $\frac{\gamma}{\lambda}=0.00001$ and $\frac{\gamma}{\lambda}=0.3$

\section{5 conclusion}

In this article we have studied a system of a two-level atom interacting resonantly with a single-mode quantized 
electromagnetic field through intensity dependent coupling. The atoms and the field are initially prepared in the excited and coherent state, respectively. The effect of atomic damping on entanglement dynamics has been discussed through some measures such as atomic QFI , atomic von Neumman entropy and negativity, von Neumman entropy for global system have been treated. . We have made a comparison between QFI and atomic von Neumman entropy as a measure of entanglement, we have shown that information about atom completely disappears as time becomes very large in the case of large damping parameter. Negativity and von Neumman entropy are studied for global system under effect of atomic decay.

\section{References}

[1] E. T. Jaynes, F. W. Cummings, Proc. IEEE, 51, 89 (1963).

[2] al. et. P. Goy, Phys. Rev. Lett., 50, 1903 (1983).

[3] Gabrielse , H. Dehmelt , Phys. Rev. Lett., 55, 67 (1985).

[4] D. Meschede, et al, Phys. Rev. Lett., 54, 551 (1985).

[5] W. B. Shore, L. P. Knight, J. Mod. Opt., 40, 1195(1993); T. M. El-Shahat, S. Abdel-Khalek, M. Abdel-Aty, A. SF. Obada, J. of Mod. Opt., 50, 2013 (2003); S. AbdelKhalek, Applied Mathematics \& Information Sciences, 1, 53 (2007); M. Abdel-Aty, S. Abdel-Khalek, A. S-F Obada, Chaos, Solitons \& Fractals, 12, 2015 (2001).

[6] B. Buck, V. C. Sukumar, Phys. Lett. A, 81, 132 (1981).

[7] S. Singh, Phys. Rev. A, 25, 3206 (1982).

[8] V. C. Sukumar, B. Buck, J. Phys. A (1984 )7 877;V. Buzek, J. Mod. Opt., 36, 1151 (1989).

[9] V. Buzek, Phys. Rev. A, 39, 3196 (1989); V. Buzek, I. Jex, Quant. Opt., 2, 147 (1989).

[10] M. Sebawe Abdalla, M. M. A. Ahmed, A.-S. F. Obada, Physica A, 170, 393 (1991).

[11] S. M. Barnett, P. L. Knight, Phys. Rev. A, 33, 2444 (1986).

[12] R. R. Puri, G. S. Agarwal, Phys. Rev. A, 35, 3433 (1987).

[13] A. J. Wonderen, Phys. Rev. A, 56, 3116 (1997); S. AbdelKhalek, A. S-F. Obada, Annals of Physics, 325, 2542 (2010).

[14] J.-M. Raimond, M. Brune, S. Haroche, Rev. Mod. Phys., 73, 565 (2001).

[15] G. Lindblad,Commun. Math. Phys., 48, 119 (1976).

[16] F. Diedrich et al, Phys. Rev. Lett., 62, 403 (1989).

[17] I. Marzoli, J. I. Cirac, R. Blatt, P. Zoller, Phys. Rev. A, 49, 2771 (1994).

[18] J. Eiselt, H. Risken, Opt. Commun 72, 351 (1989); B. Daeubler et al, Phys. Rev. A, 48, 3955 (1993).

[19] V. J. A.Wonderen, Phys. Rev. A, 56, 3116 (1997) .

[20] L. F. Wei1, Yu-xi Liu1, Markus J. Storcz, Franco Nori1, Phys. Rev. A, 73, 052307 (2006); A. S-F Obada, S. AbdelKhalek, Physica A, 389, 891 (2010); A. S-F Obada, S. AbdelKhalek, A. Plastino, Physica A, 390, 525 (2011).

[21] G. J. Peixoto, C. M. Nemes, Phys. Rev. A, 59, 3918 (1999); G. J. Peixoto, C. M. Nemes, Phys. Rev. A, 69, 063812 (2004).

[22] M. S. Chumakov et al, Phys. Rev. A, 61, 033814 (2000).

[23] L. Zhou et al, Phys. Lett. A, 284, 156 (2001).

[24] X. C. Li, F. M. Fang, Chin. Phys., 12, 866 (2003); Q-C. Zhou S-N. Zhu, Chin. Phys., 14, 1147 (2005).
[25] M. Abdel-Aty, J. Phys. A: Math. Gen., 38, 8589 (2005); M. S. Abdalla, A.-S. F. Obada and M. Abdel-Aty, Ann. Phys., 318, 266 (2005); M. Abdel-Aty, M. S. Abdalla, A.-S. F. Obada J. Phys. A: Math. Gen., 34, 9129 (2011).

[26] M. Abdel-Aty, J. Mod. Opt., 50, 161 (2003); Jian-Song Zhang and Ai-Xi Chen, Quant. Phys. Lett., 1, 69 (2012); A.B. A. Mohamed, Quant. Inf. Rev., 1, 1 (2013).

[27] A.-S. F. Obada, H. A. Hessian, J. Opt. Soc. Am. B, 21, 1535 (2004).

[28] M. H. Naderi, M. Soltanolkotabi, Eur. Phys. J. D, 39, 471 (2006).

[29] A.-S. F. Obada, H. A. Hessian, A.-B. A. Mohamed, Opt. Commun, 280, 230 (2007).

[30] R. W. Rendell, A. K. Rajagopal, Phys. Rev. A, 67, 062110 (2003).

[31] H. A. Hessian, A. -B. A. Mohamed, Chin. Phys. Lett., 25, 2492 (2008).

[32] F. Diedrich, J. C. Bergquist, W. M. Itano, D. J. Wineland, Phys. Rev. Lett., 62,403 (1989).

[33] R. R. Puri, Mathematical Methods of Quantum Optics, Springer, Berlin, 252-253 (2001).

[34] I. Marzoli, J. I. Cirac, R. Blatt, P. Zoller, Phys. Rev. A., 49, 2771 (1994).

[35] D. J. S. Phoenix, L. P. Knight, Ann. Phys., 186, (1988) 381; D. J. S. Phoenix, L. P. Knight, Phys. Rev. A, 44, 6023 (1991).

[36] V. Buzek, A. V. Barranco, P. L. Knight, Phys. Rev. A, 45, 6570 (1992).

[37] A. Peres, Phys. Rev. Lett., 76, 1413 (1996).

[38] K. Zyczkowski, P. Horodecki, A. Sanpera ,M. Lewenstein, Phys. Rev. A., 58, 883 (1998).

[39] G. Vidal, J. Mod. Opt., 47, 355 (2000).

[40] K. W. Wootters, Phys. Rev. D., 23, 357 (1981).

[41] L. S. Braunstein, M. C. Caves, Phys. Rev. Lett., 72, 3439 (1994); S. Abdel-Khalek, K. Berrada, A. S.-F. Obada, Eur. Phys. J. D, 66, 1 (2012); A. S.-F. Obada, S. Abdel-Khalek, K. Berrada, M. E. Shaheen, Physica A, 392, 6624 (2013).

[42] W. C. Helstrom, Quantum Detection and Estimation Theory. Academic Press, New York,Chap., VIII, (1976).

[43] A. S. Holevo, Probabilistic and Statistical Aspect of Quantum Theory. North-Holland, Amsterdam, (1982).

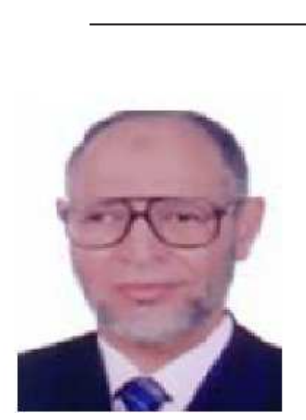

A. S. F. Obada, received D. Sc. and Ph. D. from Manchester University, 1994 and 1967. He is well known for his innovative contributions to quantum optics and became known primarily for introducing some new quantum states and some new quantum models. $\mathrm{He}$ is especially well known for profound contributions to different fields of quantum optics and quantum information, highly non-classical light, practical information security, and implementations of quantum information tasks. His current research interests include quantum resources, optical and atomic implementations of quantum information tasks and generations of new 
quantum states. Membership of Scientific Societies include : Fellow of the Institute of Physics (London), Associate of International Center for Theoretical Physics (ICTP) Trieste, Italy, and Member of the Mathematical and Physical Society of Egypt. He is the Chairman of Egyptian Mathematical Society, member of American Mathematical Society, Egyptian Academy of Sciences, and African Academy of Sciences. He has published over 200 papers in international refereed journals, some books and book chapters.

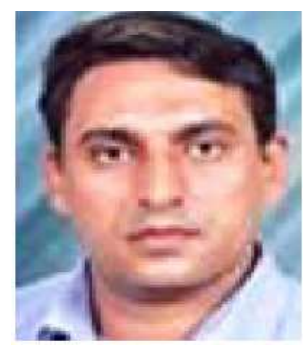

S. Abdel-Khalek has obtained his $\mathrm{PhD}$ degree in Quantum information in 2004 from Azhar University. His research interests include different directions in quantum information processing. $\mathrm{He}$ is the author of several articles published in different international scientific journals and is a member of different working groups. He is Assistant Professor, of Applied Mathematics, Mathematics Department, Faculty of Science, Sohag University, Egypt. He is presently employed as Assistant Professor of Mathematics and Statistics Department, Faculty of Science, Taif University, KSA.
M. E. Shaheen, received

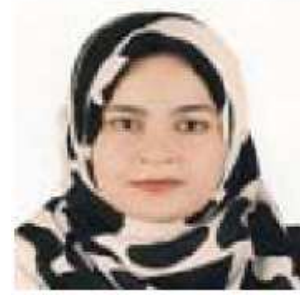

M.Sc. in theoretical mechanics from Faculty of Science, Mansoura University, Egypt. She is a student of $\mathrm{PhD}$ in Quantum Optics and assistant lecture in Mathematics department, faculty of science, Port Said University, Egypt. Brief statement of research interests: Quantum Optics and Quantum information. 